Z. Klin. Chem. Klin. Biochem.

12. Jg. 1974, S. 5-13

\title{
Die Bestimmung der Glucose in Plasma und Serum (Hexokinase/Glucose-6-Phosphat-Dehydrogenase- Methode) mit dem Greiner Electronic Selective Analyzer GSA II
}

\author{
Von R. Richterich $\dagger$, H. Küffer, Erica Lorenz und J. P. Colombo \\ Chemisches Zentrallabor, Inselspital Bern, Schweiz
}

(Eingegangen am 5. Oktober/26. November 1973)

1. Die Hexokinase/Glucose-6-Phosphat-Dehydrogenase-Methode wurde für den Greiner Elcctronic Selective Analyzer GSA II adaptiert. Durch Weglassen der Enzyme im Leerwert-Ansatz wird ein Proben- und partieller Reagenzien-Leerwert mitgeführt.

2. Der lineare Meßbereich liegt $\mathrm{zwischen} 0$ und $500 \mathrm{mg} / 100 \mathrm{ml}(0-28 \mathrm{mmol} / \mathrm{l})$.

3. Eine Verschleppung ist bei einem Konzentrations-Gefälle von 200 auf $12 \mathrm{mg} / 100 \mathrm{ml}$ nicht nachweisbar.

4. Alle untcrsuchten potentiellen Stör-Faktoren, inklusive Lipämie, Hämolyse, Bilirubin, Fructose und Mannose gaben negative Resultate.

5. Die Präzision ist eine Funktion der Absorbanz: der Variations-Koeffizient fällt von unendlich bei einer Absorbanz von Null auf $2 \%$ bei einem $A$ von $0,05,1 \%$ bei einem $A$ von 0,10 und stabilisiert sich auf $0,5 \%$ bei einer Absorbanz über 0,20 .

6. Die Konstanz der Volumina, Zeiten und Temperaturen erlaubt eine Absolut-Messung ohne Eichung über mehr als 8 Monate (bisherige Erfahrung). Die Stabilität der Reagenzien ist ausgezeichnet.

7. Die Reproduzierbarkeit ist gleich der Repetierbarkeit und ist durch die apparative und methodische Präzision gegeben.

\section{The determination of glucose in plasma and serum (hexokinase/glucose-6-phosphate dehydrogenase method) with the Greiner Electronic Selective Analyzer GSA II}

1. The hexokinase/glucose-6-phosphate dehydrogenase method was adapted for the Greiner Electronic Selective Analyzer GSA II. A sample blank and a partial reagent blank were performed by omitting the enzymes in the reagent control sample.

2. The assay is linear between 0 and $500 \mathrm{mg} / 100 \mathrm{ml}(0-28 \mathrm{mmol} / \mathrm{l})$.

3. Carry over could not be detected after a change in concentration from 200 to $12 \mathrm{mg} / 100 \mathrm{ml}$ between neighbouring samples.

4. All the investigated potential sources of interference, including lipaemia, haemolysis, bilirubin, fructose and mannose, had no effect.

5. The precision is a function of absorbance: the variation coefficient falls from infinity at an absorbance of zero, to $2 \%$ at an absorbance of $0.05,1 \%$ at 0.1 , and it stabilizes at $0.5 \%$ at absorbances greater than 0.20 .

6. With controlled constant volumes, times and temperatures, absolute determinations can be performed over 8 months (based on experience so far) without calibration.

7. The reproducibility is the same as repeatability, and is a function of the apparative and methodical precision.

Die Hexokinase $\left.{ }^{1}\right) /$ Glucose-6-Phosphat-Dehydrogenase- $^{2}$ ) Methode (Hexokinase-Methode) gilt heute als ReferenzMethode für die Bestimmung der Glucose in biologischen Flüssigkeiten. Das Prinzip dieses Verfahrens wurde zuerst von Cori et al. (1) zur Messiung der Hexokinase-Aktivität verwendet. Die ersten Glucose-Bestimmungen führten Hohorst et al. (2) in Leber-Gewebe durch. Schmidt (3) verwendete das Verfahren in der Klinik zur gleichzeitigen Messung der Glucose- und FructoseKonzentration im Serum. Czok und Bartelmai (4) führten die ersten Routine-Bestimmungen vion Blut, Liquor und Urin nach Enteiweißung mit Perchlorșäure durch. Spätere Modifikationen erlaubten eine direkte Verwendung von verdünntem Hämolysat (5-7). In unseren Laboratorien wird die Glucose seit über drei Jahren direkt, d. h. ohne vorgängige Enteiweißung mit der

1) EC 2.7.1.1

2) EC 1.1.1.49
Hexokinase-Methode bestimmt $(8,9)$. Die Methode hat sich auch als besonders geeignet für die Bestimmung der Glucose im Urin erwiesen (10-15).

Die Hexokinase-Methode wurde zuerst von Scherstén und Tibbling (16) zur Glucose-Bestimmung im Urin mechanisiert: sie führten die Bestimmung fluorometrisch auf einem AutoAnalyzer (Technicon) durch. Photometrische Adaptationen für den AutoAnalyzer wurden von Harding und Heinzel (17), Yee (18) und Neeley (19) beschrieben. Vor kurzem berichteten Haeckel und Haeckel (20) über eine Adaptation der Methode furr den Eppendorf 5030 Automaten zur Bestimmung der Glucose in enteiweißtem Blut. Schmidt et al. (21) verwendeten das Verfahren im AnalysenAutomaten C4B (Perkin-Elmer).

Wir berichten in der vorliegenden Arbeit über die Adaptation einer Hexokinase-Methode für den Greiner Electronic Selective Analyzer GSA II (GSA II) (22). Diese Methode eignet sich vor allem für die Bestim- 
mung der Glucose in Plasma, Serum, Liquor und Urin. In einer späteren Arbeit werden wir eine Modifikation beschreiben, die für die Bestimmung der Glucose in Kapillar-Blut und Kapillar-Plasma besonders geeignet ist.

\section{Material und Methoden}

Chemikalien

Äthylendiamin-tetraessigsäure, Dinatrium-Salz, Di-Hydrat (EDTA) (puriss., Fluka Nr. 03670)

Ammoniumsulfat (puriss., p. a., Fluka Nr. 09980)

ATP, Dinatrium-Salz, Tri-Hydrat (Boehringer Nr. 15028, l-g-Packung)

Glucose (Standard Reference Material, National Bureau of Standards, purity $99,9 \%$ )

Glucose-6-Phosphat-Dehydrogenase (etwa $170 \mathrm{U} / \mathrm{mg}$,

Suspension in 3,2 mol/l Ammoniumsulfat-Lösung,

Boehringer Nr. 15303, 5-mg-Packung)

Hexokinase (etwa $140 \mathrm{U} / \mathrm{mg}$, Suspension in $3,2 \mathrm{~mol} / \mathrm{l}$

Ammoniumsulfat-Lösung, Boehringer Nr. 15431, 10-mg-

Packung)

Magnesiumsulfat, Hepta-Hydrat (puriss., p. a., Fluka

Nr. 63140)

NADP, Dinatrium-Salz (Boehringer Nr. 15600, 500-mg-

Packung)

Nicotinsäureamid (pur., Fluka Nr. 72340)

Triäthanolamin-hydrochlorid (puriss., p. a., Fluka Nr 90290)

\section{Stamm-Lösungen}

Puffer-Lösung

(Konzentrationen in mmol/1: EDTA 10, Magnesiumsulfat 40,6, Nicotinsäureamid 9,83, Triäthanolamin-Salzsäure-Puffer $600, \mathrm{pH} 7,5): 55,7 \mathrm{~g}$ Triäthanolamin-hydrochlorid und $1,86 \mathrm{~g}$ EDTA werden mit demineralisiertem Wasser auf etwa $400 \mathrm{ml}$ gelöst. $5,0 \mathrm{~g}$ Magnesiumsulfat und $600 \mathrm{mg}$ Nicotinsäureamid zugeben und lösen. $\mathrm{pH}$ auf 7,5 einstellen und mit demineralisiertem Wasser auf $500 \mathrm{ml}$ auffüllen. Bei $4^{\circ} \mathrm{C}$ mindestens $1 \mathrm{Jahr}$ haltbar.

\section{Ammoniumsulfat-Lösung}

(Ammoniumsulfat $250 \mathrm{mmol} / \mathrm{l}$ ): $33 \mathrm{~g}$ Ammonium-Sulfat in etwa $800 \mathrm{ml}$ demineralisiertem Wasser lösen und auf 1 Liter auffüllen. Unbeschränkt haltbar.

Gebrauchs-Lösungen

R-1: Substrat-Reagenz

(Konzentrationen, mmol/1: ATP 1,65, EDTA 5,0, MagnesiumSulfat 20,3, NADP 0,635, Nicotinsäureamid 4,91, Triäthanolamin-Salzsäure-Puffer 300, pH 7,5): 1.g ATP (1 Packung) und $500 \mathrm{mg}$ NADP (1 Packung) in $500 \mathrm{ml}$ Puffer-Lösung lösen und mit demineralisiertem Wasser auf 1 Liter auffüllen. Im GSA II mindestens 1 Monat haltbar.

\section{R-2: Enzym-Reagenz}

(Konzentrationen, mmol/1: Ammonium-Sulfat 250, Hexokinase $50 \mathrm{mg} / \mathrm{l}(>700 \mathrm{U} / \mathrm{l})$, Hexokinase $200 \mathrm{mg} / 1$ ( $>28000 \mathrm{U} / \mathrm{l})$ ). $20 \mathrm{mg}$ Hexokinase (2 Packungen) und $5 \mathrm{mg}$ Glucose-6-Phosphat-Dehydrogenase (1 Packung) werden mit Ammoniumsulfat-Lösung auf $100 \mathrm{ml}$ gelöst. Im GSA II mindestens 1 Monat haltbar.

\section{R-3: Leerwert-Reagenz}

(Konzentration: Ammoniumsulfat $250 \mathrm{mmol} / \mathrm{l}$ ). Ammoniumsulfat-Lösung direkt verwenden. Im GSA II mindestens 1 Monat haltbar.

\section{Bestimmung der Glucose-Konzentration mit dem GSA II}

Alle vorliegenden Untersuchungen wurden mit einem Serienmodell des GSA II (22-24) durchgeführt.

\section{Beschreibung der Methode C GL 1 .}

Die Abkürzung C GL 1 steht für: Chemie, Glucose, Methode 1 (25). Das Vorgehen bei dieser Methode ist schematisch in Abbildung 1 dargestellt. Beim GSA II werden stets zwei Ansätze, ein Proben-Ansatz (S, sample assay) und ein Leerwert-Ansatz (B, blank assay) in einem Paar von Prozeß-Gefäßen (I und II) durchgeführt. $\mathrm{Da}$ aus programmatischen Gründen die Absorbanz in Ansatz I kleiner als in Ansatz II sein muß und bei der Hexokinase-Methode eine AbsorbanzZunahme erfolgt, wird Ansatz I als Leerwert (B) und Ansatz II als Proben-Ansatz (S) gewählt. Bei der vorliegenden Methode wird ein Proben- und partieller Reagenzien-Leerwert durchgeführt, d. h. es werden im Leerwert-Ansatz (Prozeß-Gefäß I) die Enzyme weggelașsen. Die einzelnen Reagenz-Volumina und dàs Timing der Reagenzien-Zugabe sind in Abbildung 1 dargestellt.

\begin{tabular}{|c|l|c|c|c|c|c|}
\hline \multicolumn{2}{|c|}{ REAGENT } & \multicolumn{2}{c|}{ DISPENSER } & \multicolumn{2}{c|}{ VOLUME } & TUBE \\
\cline { 2 - 6 } & sec & $n r$ & 1 & 11 \\
\hline & S+DF (20+100) & $594 / 600$ & $99 / 100$ & 120 & 120 \\
\cline { 2 - 7 } & DF (120) & 582 & 97 & - & - \\
\hline R-1 & Substrate Reagent & 528 & 88 & 1600 & 1600 \\
\hline R-2 & Enzyme Reagent & 498 & 83 & - & 200 \\
\hline R-4 & Blank Reagent & 336 & 56 & 200 & - \\
\hline
\end{tabular}

Fig. 1. Beschreibung der Methode C GL 1.

\section{Methoden-Programmierung}

Abbildung 2 zeigt schematisch die Methoden-Programmierung auf dem Programm-Panel. Eine ausführliche Erläuterung haben wir bereits früher gegeben $(22,26)$.

\section{Meß-Bedingungen}

Im Ansatz I (B) liegen folgende End-Konzentrationen vor (mmol/1): Ammonium-Sulfat 26,0, ATP 1,38, EDTA 4,17, Magnesiumsulfat 16,9, NADP 0,529, Nicotinsäureamid 4,09, Triäthanolamin-Salzsäure-Puffer 250, pH 7,5. In Ansatz II (S) kommen zusätzlich dazu: Hexokinase $5,20 \mathrm{mg} / 1(>700 \mathrm{U} / 1)$ und Hexokinase $20,0 \mathrm{mg} / 1(>2800 \mathrm{U} / \mathrm{l})$. Die Glucose-Konzentration im linearen Meß-Bereich liegt $z$ wischen 0 und $0,230 \mathrm{mmol} / 1$. Die Minimal-Zeiten für die einzelnen Reaktions-Schritte betragen: R-1 > 324 s (z. B. 534 s), R-2 > 300 s (z. B. 498 s) und R-3 $>48$ s (z. B. 336 s).

\section{Konstanten}

$\epsilon(\mathrm{NADPH}, 366 \mathrm{~nm})$

MG (Glucose)

$=3300 \mathrm{~cm}^{2} / \mathrm{mmol}$

$=180,16$

Proben-Volumen (SV, sample volume) $=20 \mu \mathrm{l}$ End-Volumen (FV, final volume) $\quad=1920 \mu \mathrm{l}$ 


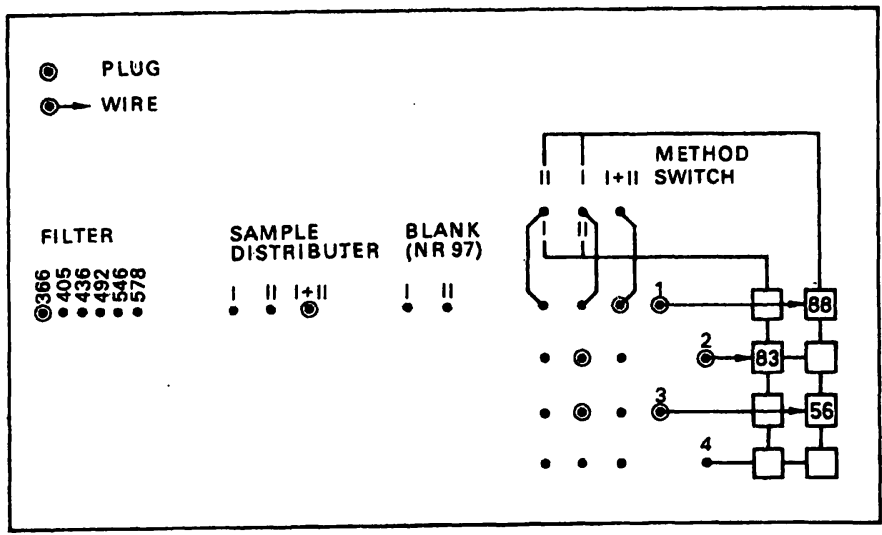

Fig. 2. Programmierung der Methode C GL 1.

\section{Berechnungen}

1. S. I. Einheit

$$
\begin{aligned}
& \mathrm{c}=\mathrm{dA} \cdot \frac{1}{\epsilon} \cdot \frac{\mathrm{FV}}{\mathrm{SV}} \cdot 1000 \mathrm{mmol} / 1 \\
& \mathrm{c}=\mathrm{dA} \cdot 29,09 \\
& \mathrm{k} \quad \text { (Methoden-Print })=2909 \\
& \text { n } \quad(\text { Zahl der Stellen nach dem Komma })=2
\end{aligned}
$$

2. Konventionelle Einheit

$$
\begin{aligned}
& c=d A \cdot \frac{1}{\epsilon} \cdot \frac{M G}{10} \cdot \frac{F V}{S V} \cdot 1000 \mathrm{mg} / 100 \mathrm{ml} \\
& c=d A \cdot 524 \mathrm{mg} / 100 \mathrm{ml} \\
& k=0524 \\
& n=0
\end{aligned}
$$

\section{Resultate und Diskussionen}

\section{Zur Reaktions-Kinetik}

Bei enzymatischen Methoden der vorliegenden Art ist der lineare Meß-Bereich durch drei Faktoren-Gruppen bestimmt:

1. der Konzentration und den $\mathrm{K}_{\mathrm{m}}$ der verwendeten Hilfs- und Indikator-Enzyme,

2. der Konzentration der Substrate und Coènzyme, d. h. im vorliegenden Fall des ATP und des NADP und

3. den Reaktions-Bedingungen, d. h. der InkubationsDauer und der Inkubations-Temperatur.

Maßgebend für die Wahl der Meß-Bedingungen ist der gewünschte lineare Meß-Bereich. Wir setzten uns zum Ziel die Methode so zu konzipieren, daß bis zu einer Glucose-Konzentration von $500 \mathrm{mg} / 100 \mathrm{ml}$ eine lineare Beziehung zwischen Absorbanz und Konzentration vorliegt. Von den oben angeführten Faktoren sind der $\mathrm{K}_{\mathrm{m}}$ der Hexokinase und der Glucose-6-Phosphat-Dehydrogenase gegeben. Die beim.GSA II vorliegende InkubationsTemperatur von $37^{\circ} \mathrm{C}$ erlaubt einen raschen Reaktionsablauf. Die für den gewünschten Meß-Bereich optimalen Enzym- und ATP-Konzentrationen wurden bereits

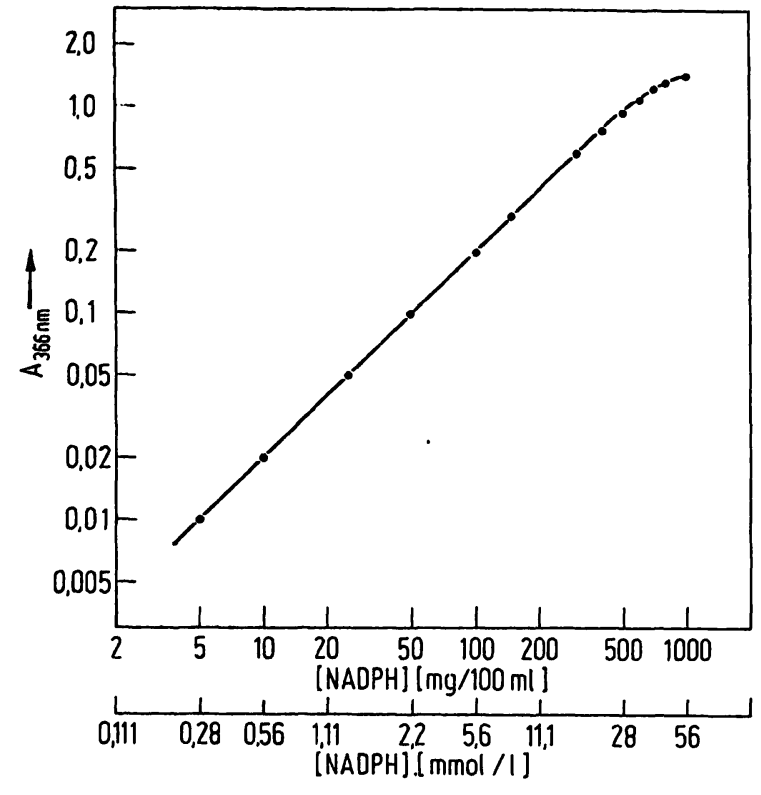

Fig. 3. Linearer Meß-Bereich bei der Bestimmung der Glucose. Die obere Grenze der Linearität ist durch die Konzentration des NADPH limitiert.

früher für eine ähnliche manuelle Methode ermittelt $(8,15)$ und für den GSA II übernommen. Unter diesen Bedingungen wird die Konzentration des NADP zum limitierenden Faktor. Wir wählten beim NADP eine End-Konzentration von $0,673 \mathrm{mmol} / \mathrm{l}$. Da die Probe im Ansatz 96fach verdünnt vorliegt, entspricht dies einer theoretisch maximal meßbaren Glucose-Konzentration von $65 \mathrm{mmol} / \mathrm{l}$ (1170 mg/100 ml) (Abb. 3). Die praktische Erfahrung hat gezeigt, daß unter diesen Voraussetzungen bei einer Inkubationsdauer über 6 min eine lineare Beziehung zwischen Absorbanz und Konzentration vorliegt und zwar bis etwa die Hälfte des vorhandenen NADP umgesetzt ist.

Liegt der Großteil der Resultate bei den Glucose-Bestimmungen unter $250 \mathrm{mg} / 100 \mathrm{ml}$, so können die NADP- und Enzym-Konzentrationen auf die Hälfte reduziert werden. Dies mag z. B. bei Screening-Untersuchungen zweckmäßig sein, bei denen eine Linearität bis zu $250 \mathrm{mg} / 100 \mathrm{ml}$ vollauf genügt. In jedem Fall kann durch eine Vorverdünnung von $1+9$ der Linearitäts-Bereich um einen Faktor von 10 vergrößert werden.

Optische Eigenschaften des Reaktions-Produktes

Für die folgenden Untersuchungen wurde ReferenzMaterial des National Bureau of Standards verwendet. Zum Vergleich wurden auch kommerzielle EichLösungen (Preciset, Boehringer) mitgeführt.

Eine typische Versuchsreihe ist in Tabelle 1 dargestellt. Es liegt eine lineare Beziehung zwischen Absorbanz und Glucose-Konzentration im Bereich von Null bis $500 \mathrm{mg} / 100 \mathrm{ml}$ (etwa 27,5 mmol/l) vor. Eine Berechnung der Regression in diesem Meßbereich ergab

$$
c=0,0014+29,090 \mathrm{dA} \mathrm{mmol} / \mathrm{l} \text {. }
$$


Tab. 1. Bestimmung der Glucose-Konzentration in wäßrigen Lösungen. Bei jeder Konzentration wurden 10 Bestimmungen durchgeführt

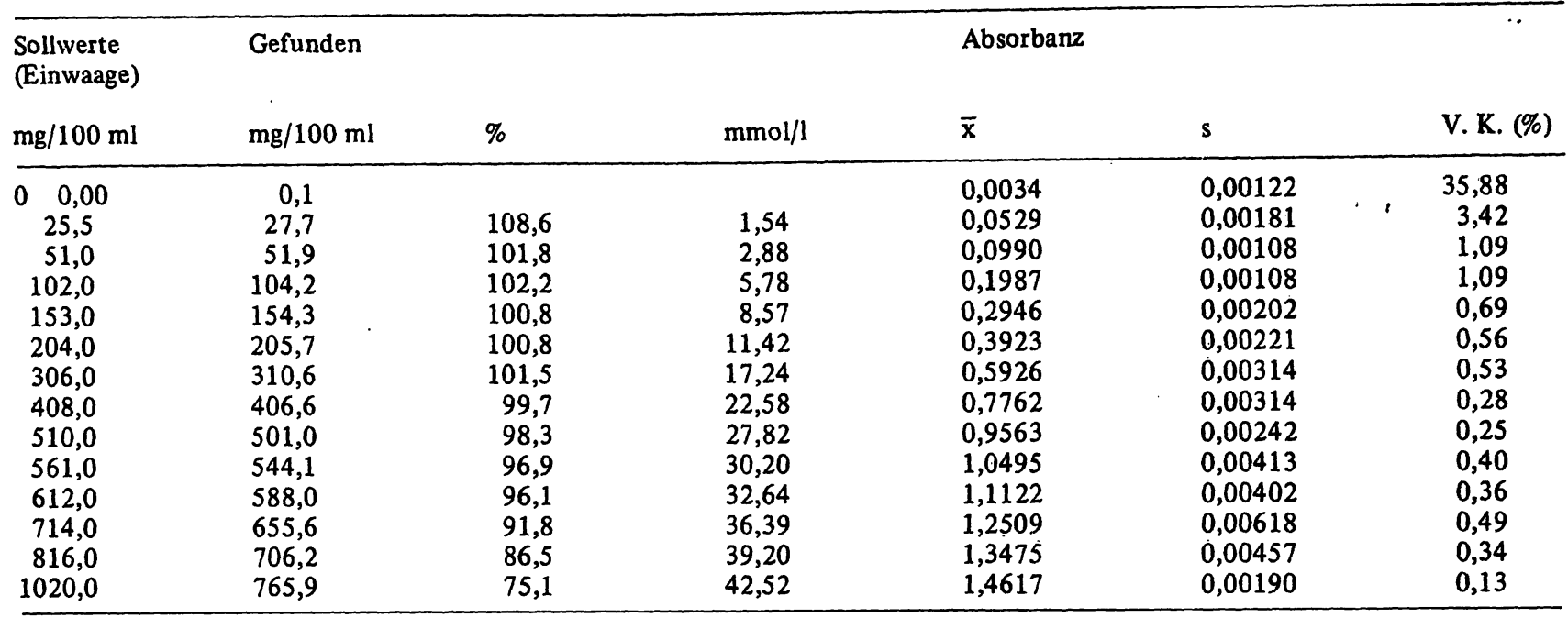

Die Standard-Abweichung betrug $0,00270 \mathrm{mg} / 100 \mathrm{ml}$, der Korrelations-Koeffizient 1,0 (6 Stellen). a weicht nicht von Null ab. Die Größe von b ist im vorliegenden Fall mit demjenigen des Methoden-Faktors $(29,09)$ identisch. Die Ursache der bei diesem Versuch systematischen Abweichung bei den tiefen Glucose-Konzentrationen konnten wir nicht eruieren. Es handelt sich dabei nicht um eine konstante Beobachtung. Ähnliche Erfahrungen machten wir auch mit Preciset (Boehringer). Die gefundenen Werte lagen hier zwischen 99,7 und 101,7\%, im Mittel 100,63\% der deklarierten Werte.

Bei der Entwicklung dieser Methode gingen wir von einer molaren Absorptivität des NADPH bei $366 \mathrm{~nm}$ von $3300 \mathrm{~cm}^{2} / \mathrm{mmol}$ aus (27-29).

Tab. 2. Bestimmung der Glucose-Konzentration in Preciset (Boehringer) Resultate in $\mathrm{mg} / 100 \mathrm{ml}(\mathrm{N}=10)$

\begin{tabular}{lrrrr}
\hline Sollwerte & \multicolumn{3}{l}{ Gefundene Werte } & \\
& $\overline{\mathbf{X}}$ & $\%$ & s & V. K. \\
\hline 50 & 50,20 & 100,4 & 1,135 & 2,26 \\
100 & 101,70 & 101,7 & 1,767 & 1,74 \\
150 & 149,50 & 99,7 & 0,850 & 0,57 \\
200 & 202,60 & 101,3 & 1,506 & 0,74 \\
300 & 302,20 & 100,7 & 1,398 & 0,46 \\
400 & 400,00 & 100,0 & 1,633 & 0,41 \\
\hline
\end{tabular}

Auf Grund unserer Erfahrungen stimmen die durch Einwägen erhaltenen Werte sehr gut mit diesen Angaben in der Literatur überein. Die von zwei Seiten $(7,20)$ geäußerten Kritiken, wonach mit dieser molaren $\mathrm{Ab}$ sorptivität im Vergleich zu derjenigen bei $334 \mathrm{~nm}$ um 3-4\% zu hohe Werte erhalten werden, können wir nicht bestätigen; wir vermuten vielmehr, daß optische Faktoren (Streulicht?) für die beobachteten Diskrepanzen verantwortlich waren. Es scheint uns jedoch außerordentlich wichtig, daß diese Unstimmigkeiten geklärt werden, da die molare Absorptivität des NADPH ein Basiswert der Enzymologie und Klinischen Chemie ist.

\section{Zur Wahl der Leerwerte}

Bei korrekter Durchführung der manuellen HexokinaseMethode muß entweder enteiweißt (4) oder aber ein Proben- und partieller Reagenzien-Leerwert mitgeführt werden $(8,15)$. Von einer direkten Bestimmung ohne Leerwert in Plasma oder Serum ist auf jeden Fall abzuraten, da sich im Plasma zahlreiche im Uitravioletten absorbierende Substanzen finden. Selbst die Verwendung der üblichen Enteiweißungsmittel, Perchlorsäure und Trichloressigsäure, scheint heute fraglich geworden zu sein (20), da sich offenbar die durch diese Säuren nicht präzipitierten Glycoproteine (Seromucoid, $\alpha$ Glycoprotein, Haptoglobine) störend auswirken können.

Bei den bisher gebräuchlichen Analysatoren ist das Leerwert-Problem unterschiedlich gelöst. Beim AutoAnalyzer werden offenbar durch die Dialyse der größte Teil der interferierenden Substanzen eliminiert $(18,19)$. Für den C 4 B (Perkin-Elmer) wird die Verwendung eines Leerwert-Kanals empfohlen (21). Beim EndpunktAutomaten 5030 (Eppendorf) müssen entweder die Leerwerte separat analysiert werden oder aber eine Enteiweißung mit Zinkhydroxid vorgenommen werden (20).

Das Konzept des GSA II fordert bei jeder Bestimmung das Mitführen eines Leerwertes. Im vorliegenden Fall drängt sich - analog der manuellen Methode (8) - das Weglassen der Hexokinase und der Glucose-6-PhosphatDehydrogenase bei sonst identischen Konzentrationen im Leerwert-Ansatz auf. Diese beiden Enzyme weisen in den eingesetzten Konzentrationen bei $366 \mathrm{~nm}$ keine Absorbanz auf. Wir führen somit einen Proben- und partiellen Reagenzien=Leerwert durch.

\section{Spezifität}

\section{Selektivität}

Theoretisch ist die Hexokinase-Methode absolut selektiv. Da neben Glucose aber auch Fructose und Mannose 
durch Hexokinase phosphoryliert werden, besteht die Gefahr, daß bei der Verwendung unreiner Enzyme diese Hexose-Phosphate durch die entsprechenden Isomerasen in Glucose-6-Phosphat umgewandelt werden $(3,12-14)$. Solchen theoretischen Erwägungen, die offenbar auf der Verwendung relativ unreiner Enzyme beruhen, stehen aber die praktischen Erfahrungen gegenüber. Nur bei sehr hohen Fructose-Konzentrationen soll tatsächlich mit einer Störung zu rechnen sein: so sollen $360 \mathrm{mg} / 100 \mathrm{ml}(20 \mathrm{mmol} / \mathrm{l})$ Fructose eine scheinbare Glucose-Konzentration von $9,5 \mathrm{mg} / 100 \mathrm{ml}$ $(0,53 \mathrm{mmol} / \mathrm{l})$ vortäuschen. Von anderer Seite $(13,14)$ wurde bei hohen Fructose-Konzentrationen nicht eine positive, sondern eine negative Interferenz beobachtet, die durch eine Kompetition um die Hexokinase gedeutet wurde.

$\mathrm{Da}$ die Möglichkeit einer Interferenz vor allem von der Reinheit der Enzyme, sowie der Konzentration der Enzyme, des NADP und des ATP's abhängig ist, führten wir einige Experimente durch. Es wurden je eine wäßrige Lösung von Glucose mit etwa $100 \mathrm{mg} / 100 \mathrm{ml}$ und Fructose, bzw. Mannose mit je $10 \mathrm{~g} / 100 \mathrm{ml}$ hergestellt. Durch Misch-Versuche wurden der Einfluß der Fructose und der Mannose auf die Messung der vorliegenden Glucose untersucht. Die Resultate dieser Versuche sind in Tabelle 3 zusammengestellt. Aus diesen Untersuchungen geht hervor, daß unter den vorliegenden Bedingungen Fructose in Konzentrationen über $\mathrm{lg} / 100 \mathrm{ml}$ einen geringen positiven Effekt hat, der aber selbst bei $5 \mathrm{~g} / 100 \mathrm{ml}$ den gemessenen Glucose-Wert um weniger als $10 \%$ erhöht. Bei der Mannose ließ sich bis zu Konzentrationen von $5 \mathrm{~g} / 100 \mathrm{ml}$ überhaupt kein Einfluß nachweisen. Die Hexokinase-Methode muß daher in der vorliegenden Modifikation als absolut selektiv gelten.

Tab. 3. Einfluß von Fructose und Mannose auf die Glucose-Bcstimmung $(\mathrm{N}=6)$. Alle Resultate in $\mathrm{mg} / 100 \mathrm{ml}$. Die Fructose und Mannose wurde in verschiedenen Mengen in das gleiche Serum eingewogen.

\begin{tabular}{lllll}
\hline $\begin{array}{l}\text { Zusatz } \\
\text { (Fructose, } \\
\text { bzw. } \\
\text { Mannose) }\end{array}$ & \multicolumn{2}{l}{ Glucose-Konzentration } \\
& $\overline{\mathrm{X}}$ & $\mathrm{s}$ & $\overline{\mathrm{X}}$ & $\mathrm{s}$ \\
\hline 0 & 96,2 & 0,79 & 95,6 & 1,43 \\
500 & 96,3 & 1,25 & 96,4 & 1,30 \\
1000 & 96,8 & 1,93 & 96,1 & 0,99 \\
2000 & 99,6 & 1,26 & 96,5 & 1,08 \\
3000 & 100,4 & 0,97 & 97,5 & 0,97 \\
4000 & 102,4 & 0,97 & 97,2 & 1,55 \\
5000 & 104,2 & 0,92 & 98,9 & 0,99 \\
\hline
\end{tabular}

\section{Chemische Interferenzen}

Uber potentielle chemische Stör-Faktoren bei der Hexokinase-Methode liegen mehrere Untersuchungen vor $(4,5,12-15,18-20)$. Außer den erwähnten fragwürdigen Störungen durch Fructose und Mannose geben alle Untersuchungen Zucker (Arabinose, Ascorbinsäure,
Dextrane, Galactose, Glucuronsäure, Inositol, Lactose, Maltose, Mannose, Rhamnose, Ribose, Saccharose, Xylose u. a.) keine Störungen. Ebenfalls negativ verliefen Interferenz-Versuche mit verschiedenen Metaboliten (Creatinin, Fumarsäure, Glutathion, Harnsäure, Phosphat $u$. a.) und Medikamenten ( $p$-AminoHippurat, Ampicillin, L-DOPA, Tetracycline, Tolazamid, Tolbutamid, u. a.). Negative Resultate wurden auch mit den üblichen Glycolyse-Hemmern (Fluorid, Monojodacetat) und Antikoagulantien (Heparin, Oxalat, EDTA) erhalten.

Das Fehlen eines Einflusses einer Hämolyse $(18,19)$ schien uns überprüfenswert, da immerhin damit gerechnet werden könnte, daß das entstehende 6-Phospho-Gluconat durch ery throcy täre 6-Phospho-Gluconat-Dehydrogenase ${ }^{3}$ ) weiter umgesetzt wird (6). Ein Zusatz von frisch hergestelltem Hämolysat zu einem glucose-haltigen Plasma ergab jedoch, wie aus Tabelle 4 hervorgeht, keine Beeinflussung des gemessenen Resultates. Auch mit Bilirubin (Tab. 5) konnte, wie bereits früher beobacht't wurde $(18,19)$, keine Störung beobachtet werden.

Tab. 4. Einfluß cincr Hämolyse auf die Glucose-Bestimmung $(\mathrm{N}=5)$

\begin{tabular}{llllll}
\hline $\begin{array}{l}\text { Hämoglobin- } \\
\text { Konzentration } \\
\text { mmol/l }\end{array}$ & $\mathrm{mg} / 100 \mathrm{ml}$ & \multicolumn{3}{l}{ Glucose-Konzentration } \\
& & $\mathrm{mmol} / \mathrm{l}$ & & \multicolumn{3}{l}{$\mathrm{mg} / 100 \mathrm{ml}$} \\
& & $\overline{\mathrm{X}}$ & $\mathrm{s}$ & $\mathrm{V} . \mathrm{K} .(\%)$ \\
\hline 0 & 0 & 6,306 & 0,0633 & 1,004 & 113,6 \\
12,4 & 20 & 6,317 & 0,8367 & 0,046 & 113,8 \\
19,9 & 32 & 6,284 & 0,0464 & 0,739 & 113,2 \\
24,8 & 40 & 6,317 & 0,0464 & 0,735 & 113,8 \\
47,2 & 76 & 6,273 & 0,0393 & 0,626 & 113,0 \\
70,1 & 113 & 6,306 & 0,0633 & 1,004 & 113,6 \\
93,7 & 151 & 6,273 & 0,0556 & 0,885 & 113,0 \\
117 & 188 & 6,273 & 0,0393 & 0,626 & 113,0 \\
174 & 281 & 6,217 & 0,0607 & 1,094 & 112,0 \\
231 & 373 & 6,239 & 0,1082 & 1,949 & 112,4 \\
287 & 463 & 6,134 & 0,1902 & 3,100 & 110,5 \\
562 & 905 & 5,928 & 0,2199 & 3,710 & 106,8 \\
\hline
\end{tabular}

Tab. 5. Einfluß von Bilirubin auf dic Glucose-Bestimmung $(\mathrm{N}=5)$. Das Bilirubin wurde in Carbonat-Lösung aufgelöst und cinem Serum zugesetzt.

\begin{tabular}{lllll}
\hline Bilirubin-Konzentration & \multicolumn{2}{l}{ Glucosc-Konzentration } \\
$\mathrm{mg} / 100 \mathrm{ml}$ & $\mathrm{mmol} / \mathrm{l}$ & $\mathrm{mmol} / \mathrm{l}$ & & $\mathrm{mg} / 100 \mathrm{ml}$ \\
& & $\overline{\mathrm{X}}$ & $\mathrm{s}$ & \\
\hline 0 & & & & \\
2,39 & 41,0 & 5,759 & 0,0833 & 103,8 \\
3,21 & 54,9 & 5,704 & 0,1144 & 102,8 \\
6,98 & 119,4 & 5,842 & 0,0531 & 105,3 \\
11,65 & 199,2 & 5,745 & 0,0717 & 103,5 \\
16,43 & 280,9 & 5,773 & 0,0453 & 104,0 \\
20,82 & 355,6 & 5,718 & 0,0785 & 103,0 \\
\hline
\end{tabular}

3) EC 1.1.1.44 


\section{Physikalische Interferenzen}

Es ist bekannt, daß das Vorliegen einer Lipämie selbst dann, wenn eine Enteiweißung vorgenommen wird, auch bei der Hexokinase-Methode zu beträchtlichen Fehlern führen kann (20). Wir untersuchten daher mehrere lipämische Seren vor und nach einer Klärung durch Zentrifugation während $20 \mathrm{~min}$ bei $15000 \mathrm{~g}$ (26). Einige dieser Resultate sind in Tabelle 6 zusammengestellt. Wie aus diesen Beobachtungen hervorgeht, stört das Vorliegen einer Lipämie die Glucose-Bestimmung mit dem GSA II nicht. Ebenfalls negative Resultate wurden mit Albumin (Tab. 7) erhalten. Alle diese Beobachtungen weisen auf die große Bedeutung des Mitfuhrens geeigneter Leerwerte bei mechanisierten Bestimmungen hin.

\section{Beeinflussung}

Die Möglichkeit einer Beeinflussung der Resultate durch Verschleppung wurde mit einer früher beschriebenen Versuchs-Anordnung überprüft $(25,31)$. Die Resultate dieses Experimentes sind in Tabelle 8 zusammengefaßt.

Tab. 6. Glucose-Bestimmungen in lipämischen und durch Zentrifugation geklärten Seren, $(15000 \mathrm{~g}, 20 \mathrm{~min})$

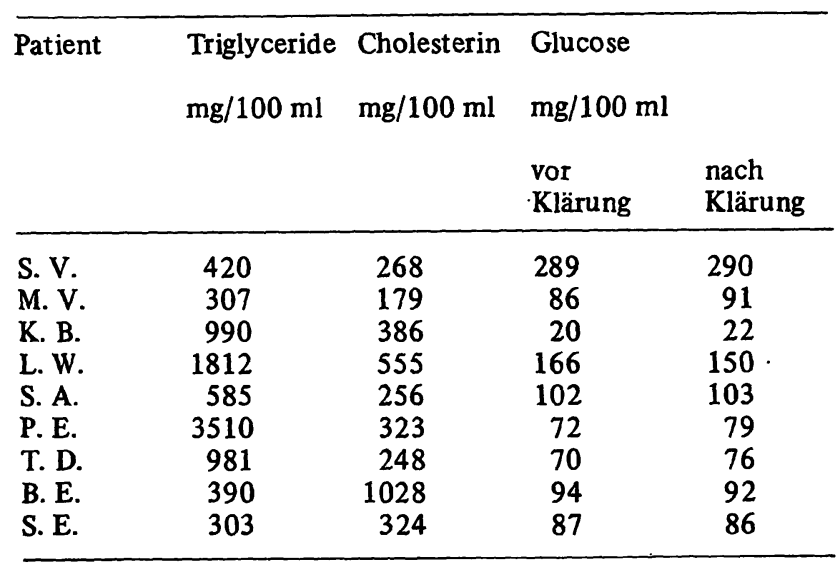

Tab. 7. Einfluß von Protein auf die Glucose-Konzentration $(\mathrm{N}=10)$. Als Ausgangs-Lösungen diente eine $20 \mathrm{~g} / \mathrm{l}$ Albumin-Lösung und eine wäßrige Glucose-Lösung $(195 \mathrm{mg} / 100 \mathrm{ml})$. Die einzelnen Ansätze wurden durch Mischung hergestellt.

\begin{tabular}{llll}
\hline $\begin{array}{l}\text { Protein- } \\
\text { Konzentration } \\
\mathrm{g} / 100 \mathrm{ml}\end{array}$ & \multicolumn{2}{l}{ Glucose-Konzentration } \\
& $\mathrm{mg} / 100 \mathrm{ml}$ & & \\
& $\overline{\mathrm{X}}$ & $\mathrm{s}$ & $\mathrm{V} . \mathrm{K} .(\%)$ \\
\hline 0 & 97,70 & 0,709 & 0,726 \\
1 & 96,10 & 1,039 & 1,081 \\
2 & 96,60 & 0,919 & 0,951 \\
3 & 97,10 & 1,000 & 1,030 \\
4 & 96,80 & 1,131 & 1,168 \\
5 & 96,20 & 1,060 & 1,102 \\
6 & 96,30 & 0,956 & 0,993 \\
7 & 96,90 & 0,824 & 0,850 \\
8 & 97,20 & 1,028 & 1,058 \\
9 & 97,00 & 0,927 & 0,956 \\
10 & 96,70 & 0,858 & 0,887 \\
\hline
\end{tabular}

Tab. 8. Versuch zum Nachweis einer Verschleppung. Es wurden 10 Versuchsreihen durchgeführt.

Die prozentuale Verschleppung (E) wurde wie folgt berechnet:

$$
E(\downarrow)=\frac{t_{1}-t_{5}}{h_{5}-t_{5}} \text { und } E(\uparrow)=\frac{h_{5}-h_{1}}{h_{5}-t_{5}}
$$

\begin{tabular}{lllllcl}
\hline Reihe & $\mathrm{t}_{1}$ & $\mathrm{t}_{5}$ & $\mathrm{~h}_{1}$ & $\mathrm{~h}_{5}$ & $\mathrm{E}(\downarrow)$ & $\mathrm{E}(\uparrow)$ \\
\hline 1 & 13,0 & 14,0 & 200,0 & 201,0 & $-0,0053$ & 0,0053 \\
2 & 12,0 & 13,0 & 197,0 & 201,0 & $-0,0053$ & 0,0213 \\
3 & 11,0 & 13,0 & 200,0 & 200,0 & $-0,0107$ & 0 \\
4 & 13,0 & 12,0 & 200,0 & 200,0 & 0,0053 & 0 \\
5 & 12,0 & 12,0 & 202,0 & 199,0 & 0 & $-0,0160$ \\
6 & 12,0 & 12,0 & 198,0 & 200,0 & 0 & 0,0106 \\
7 & 12,0 & 13,0 & 200,0 & 200,0 & $-0,0053$ & 0 \\
8 & 12,0 & 12,0 & 201,0 & 199,0 & 0 & $-0,0107$ \\
9 & 12,0 & 14,0 & 201,0 & 200,0 & $-0,0107$ & $-0,0054$ \\
10 & 12,0 & 11,0 & 202,0 & 199,0 & 0,0053 & 0,0160 \\
\hline$\overline{\mathrm{X}}$ & 12,1 & 12,6 & 200,1 & 199,9 & $-0,0027$ & 0,0011 \\
\hline
\end{tabular}

Ähnlich wie bei den Untersuchungen über die Möglichkeit einer Verschleppung bei der Harnstoff-Bestimmung (26) war es auch im vorliegenden Fall nicht möglich, eine Verschleppung bei einem Konzentrations-Gefälle von 200 auf $12 \mathrm{mg} / 100 \mathrm{ml}$ nachzuweisen.

\section{Rich tigkeit}

Die Richtigkeit wurde zunächst durch Vergleichs-Bestimmungen mit einigen komerziellen Kontroll-Seren ermittelt. Wie aus Tabelle 9 hervorgeht, war die Übereinstimmung vor allem mit den enzymatisch bestimmten Werten gut.

Als nächstes wurden je 100 echte (blinde) Doppelbestimmungen mit einer manuellen Methode $(8 ; 9)$ und dem GSA durchgeführt. Die Resultate sind in Tabelle 10 zusammengestellt. Bei einem arithmetischen Mittel von $107,3 \mathrm{mg} / 100 \mathrm{ml}$ betrug die Standard-Abweichung 2,15 mg/100 $\mathrm{ml}$ bei der manuellen Methode; dies entspricht einem Variations-Koeffizienten von $2 \%$. Beim GSA II lag das arithmetische Mittel bei $107,55 \mathrm{mg} / 100 \mathrm{ml}$, die Standard-Abweichung zwischen den Doppel-Werten bei 0,896 mg/100 ml; der Variations-Koeffizient zwischen den Mitteln der Doppel-Bestimmungen betrug 0,833\%.

Zusammen mit! den früher erwähnten Untersuchungen mit eingewogenen Substanzen bestätigen die erwähnten Beobachtungen die hohe Richtigkeit der Methode.

\section{Präzision}

In einer vorangehenden Arbeit (26) führten wir die Begriffe der apparativen und methodischen Präzision ein. Zur Beurteilung der Summe dieser beiden FehlerGruppen eignet sich die in Abbildung 4 gewählte Darstellung. Ausgewertet wurden die in Tabelle 1 angeführten Daten. Auf die Abszisse sind die Glucose-Konzentrationen, bzw. die Absorbanz bei den gewählten Konzentrationen dargestellt. Bei jeder Konzentration wurden 10 Bestimmungen durchgeführt. Die Ordinate 
Tab. 9. Bestimmung der Glucose-Konzentration in komerziellen Kontroll-Seren (Resultate in $\mathrm{mg} / 100 \mathrm{ml}, \mathrm{N}=5$ )

\begin{tabular}{|c|c|c|c|c|c|c|c|c|}
\hline \multirow[t]{2}{*}{ Serum } & \multirow[t]{2}{*}{$\left.\operatorname{Art}^{*}\right)$} & \multicolumn{4}{|l|}{ Deklarierte Werte } & \multicolumn{3}{|c|}{ Gefundene Werte } \\
\hline & & Methode & $\overline{\mathbf{x}}$ & $\pm 2 \mathrm{~s}$ & & $\overline{\mathbf{x}}$ & $\mathbf{s}$ & V. K. (\%) \\
\hline Precinorm $S^{\prime}$ ) & $\mathbf{L}$ & $\begin{array}{l}\text { HK/G-6-P-DH } \\
\text { GOD-Perid (Boehringer) } \\
\text { GOD-o-Dianisidin } \\
\text { O-Toluidin }\end{array}$ & $\begin{array}{l}106 \\
107 \\
109 \\
109\end{array}$ & $\begin{array}{l}95.4 \\
96,3 \\
98,1 \\
98.1\end{array}$ & $\begin{array}{l}4-117 \\
3-118 \\
1-120 \\
1-120\end{array}$ & 100.0 & 1,054 & 1,05 \\
\hline Patho-trol ${ }^{2}$ ) & $\mathbf{F}$ & $\begin{array}{l}\text { HK/G-6-P-DH } \\
\text { GOD-o-Dianisidin } \\
\text { o-Toluidin } \\
\text { AutoAnalyzer (N-2a) } \\
\text { Nelson-Somogyi }\end{array}$ & $\begin{array}{l}257 \\
275 \\
255 \\
272 \\
276\end{array}$ & $\begin{array}{l}245 \\
262 \\
266\end{array}$ & $\begin{array}{l}-265 \\
-282 \\
-286\end{array}$ & 248.9 & 1.235 & 0,50 \\
\hline $\begin{array}{l}\left.\text { Lab-Trol }^{2}\right) \\
(40 A-Z)\end{array}$ & $\mathbf{F}$ & $\begin{array}{l}\text { HK/G-6-P-DH } \\
\text { GOD-Perid (Boehringer) } \\
\text { GOD-O-Dianisidin } \\
\text { o-Toluidin } \\
\text { AutoAnalyzer (N-2b } 1 / 11 \text { ) } \\
\text { Nelson-Somogyi }\end{array}$ & $\begin{array}{r}93 \\
100 \\
95 \\
92 \\
100 \\
97\end{array}$ & $\begin{array}{l}87 \\
94 \\
99 \\
86 \\
92 \\
92\end{array}$ & $\begin{array}{l}-99 \\
-106 \\
-111 \\
-98 \\
-108 \\
-103\end{array}$ & 93,2 & 0,820 & 0,88 \\
\hline $\begin{array}{l}\left.\text { Moni-trol } 1^{2}\right) \\
(\text { LTD-114 A, B) }\end{array}$ & $L$ & $\begin{array}{l}\text { HK/G-6-P-DH } \\
\text { GOD-Perid (Boehringer) } \\
\text { GOD-o-Dianisidin } \\
\text { O-Toluidin } \\
\text { AutoAnalyzer (N-2b } 1 / 11) \\
\text { SMA 12/60 }\end{array}$ & $\begin{array}{r}87 \\
88 \\
91 \\
100 \\
95 \\
101\end{array}$ & $\begin{array}{l}81 \\
82 \\
85 \\
94 \\
87 \\
88\end{array}$ & $\begin{array}{l}-93 \\
-94 \\
-96 \\
-106 \\
-103 \\
-114\end{array}$ & 87,6 & 1,054 & 1,20 \\
\hline $\begin{array}{l}\text { Moni-trol } \mathrm{Il}^{2} \text { ) } \\
\text { (PTD-27 A) }\end{array}$ & $L$ & $\begin{array}{l}\text { HK/G-6-P-DH } \\
\text { GOD-Perid (Boehringer) } \\
\text { GOD-o-Dianisidin } \\
\text { o-Toluidin } \\
\text { AutoAnalyzer }(\mathrm{N}-2 \mathrm{~b} 1 / 11) \\
\text { SMA } 12 / 60\end{array}$ & $\begin{array}{l}233 \\
225 \\
255 \\
232 \\
240 \\
251\end{array}$ & $\begin{array}{l}221 \\
217 \\
239 \\
223 \\
233 \\
237\end{array}$ & $\begin{array}{l}-245 \\
-233 \\
-271 \\
-241 \\
-247 \\
-265\end{array}$ & 223,7 & 1,203 & 0,54 \\
\hline
\end{tabular}

*) F flüssig L lyophilisiert

1) Boehringer, Mannheim

2) Dade, USA

HK/G-6-P-DH = Hexokinase/Glucose-6-Phosphat-Dehydrogenase GOD $=$ Glucoseoxidase

Tab. 10. Vergleichs-Doppel-Bestimmungen mit einer manuellen Methode (Hexokinase/Glucose-6-Phosphat-Dehydrogenase und dem GSA II. Resultate in $\mathrm{mg} / 100 \mathrm{ml}$

\begin{tabular}{lcc}
\hline Methode & Manuell & GSA II \\
\hline $\mathbf{N}$ & $2 \cdot 100$ & $2 \cdot 100$ \\
$\overline{\mathbf{X}}$ & 107,258 & 107,551 \\
$\overline{\mathbf{X}}_{\text {Diff }}$ & 2,2424 & 0,9192 \\
SDiff & 2,1485 & 0,8961 \\
V. K. (\%) & 2,001 & 0,833 \\
\hline
\end{tabular}

zeigt die Standard-Abweichungen dẹ Resultate ebenfalls die Absorbanz - bei den einzelnen Konzentrationen. Es ergibt sich eine Fehler-Gerade mit der Regression

$$
s=0,00126+0,00242 \mathrm{dA},
$$

einer Standard-Abweichụng von 0,00114 und einem Korrelations-Koeffizienten von 0,71287. Die Kurven

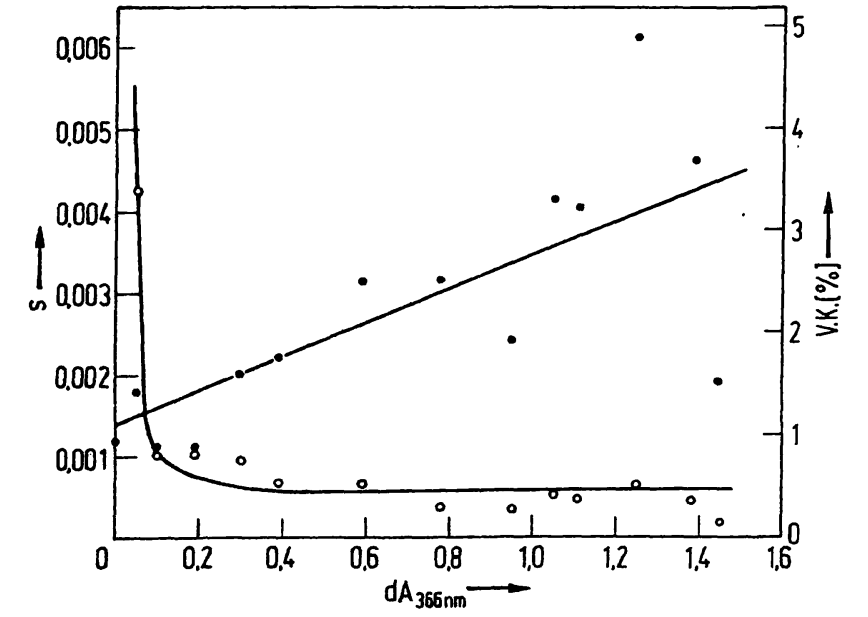

Fig. 4. Graphische Darstellung der in Tabelle 1 angefuhrten Versuche. Abszisse: Absorbanz, Ordinate: zugehörige Standard-A bweichungen $(\bullet$, links) und Variations-Koeffizienten $(0$, rechts). 
entsprechen absolut denjenigen bei der Harnstoff-Bestimmung (26). Der Variations-Koeffizient hat die höchsten Werte bei einer Absorbanz um null und fällt kontinuierlich auf Werte unter $1 \%$.

Die Repetierbarkeit der Methode geht aus mehreren der oben angefuihrten Experimente hervor. Sie ist primär eine Funktion der Absorbanz und liegt in Prozent ausgedrückt bei einer Absorbanz über 0,15 unter $1 \%$.

Die Reproduzierbarkeit wurde auf Grund einiger Daten einer Qualitäts-Kontrolle während drei Monaten ermittelt. Verwendet wurden drei Pool-Seren, die in einzelne Röhrchen abgefüllt und tief gefroren aufbewahrt wurden. Täglich wurde einmal je ein Röhrchen untersucht. Die Resultate sind in Tabelle 11 zusammengestellt. Dabei muß berücksichtigt werden, daß

1. keine Justierungen vorgenommen wurden, nachdem der GSA II im Januar 1973 mit der Initial-Justierung eingestellt worden war und

2. die Reagenzien immer nur dann nachgefüllt wurden, wenn die Füllstand-Anzeige vor einem Ausgehen warnte.

Bei tiefen Glucose-Konzentrationen (um $35 \mathrm{mg} / 100 \mathrm{ml}$ ) lag der Variations-Koeffizient um 4\%, bei mittleren Konzentrationen (um $140 \mathrm{mg} / 100 \mathrm{ml}$ ) um 1,5\% und bei hohen Konzentrationen (um 290 mg/100 ml) um 1,2\%. Versuche über die Repetierbarkeit und die Reproduzierbarkeit unterscheiden sich beim GSA II praktisch nur dadurch, daß im ersten Fall Proben aus einem einzigen Röhrchen untersucht werden während bei der Reproduzierbarkeit täglich ein anderes Röhrchen zur Untersuchung kam. Da nach den wenigen vorhandenen Angaben (31) die Unterschiede zwischen verschiedenen Specimen zur Qualitäts-Kontrolle in der Größen-Ordnung von $1 \%$ liegen, darf wohl der Schluß gezogen werden, daß die beobachteten Unterschiede zwischen Repetierbarkeit und Reproduzierbarkeit durch das Untersuchungsmaterial bedingt waren.

\section{Qualitätskontrolle und praktische Erfahrungen}

Die vorliegende Methode ist seit Januar 1973 während $24 \mathrm{~h}$ täglich im Routine-Einsatz. Nach einer anfänglichen Prüfphase wurde die Qualitätskontrolle auf ein Mini-
Tab. 11. Resultate einer Qualitäts-Kontrolle während 3 Monaten. Verwendung von 3 Kontroll-Seren. Täglich je eine $\mathrm{Be}$ stimmung in einem frisch aufgetauten Röhrchen. Alle Resultate in $\mathrm{mg} / 100 \mathrm{ml}$.

\begin{tabular}{lccc} 
& April & Mai & Juni \\
\hline Specimen I & \multicolumn{3}{c}{} \\
\hline $\mathrm{N}$ & 23 & 25 & 1 \\
$\overline{\mathrm{X}}$ & 286,0 & 293,8 & 29 \\
$\mathrm{~s}$ & 4,2 & 3,37 & 292,8 \\
V. K. (\%) & 1,47 & 1,15 & 3,88 \\
& & & 1,33
\end{tabular}

Specimen II

\begin{tabular}{lrrr}
\hline $\mathrm{N}$ & 23 & 25 & 29 \\
$\mathrm{X}$ & 35,7 & 36,0 & 36,5 \\
$\mathrm{~s}$ & 2,0 & 1,4 & 1,5 \\
V. K. (\%) & 5,6 & 3,9 & 4,0 \\
\hline
\end{tabular}

Specimen III

\begin{tabular}{lccc}
\hline $\mathrm{N}$ & 23 & 25 & 29 \\
$\overline{\mathrm{X}}$ & 141,0 & 143,8 & 144,4 \\
$\mathrm{~s}$ & 1,59 & 2,41 & 2,13 \\
V. K. (\%) & 1,13 & 1,68 & 1,48 \\
\hline
\end{tabular}

mum reduziert. Sie beschränkt sich heute auf eine täglich zweimal durchgeführte Null-Kontrolle (mit einer 0,01 mol/1 Essigsäure-Lösung) und einer Aktivitäts-Kontrolle (mit einer wäßrigen oder protein-haltigen Lösung mit einer Glucose-Konzentration um $200 \mathrm{mg} / 100 \mathrm{ml}$ ). Alle weitergehenden Überwachungen haben sich als überflüssig erwiesen. Die Stabilität der Reagenzien war ausgezeichnet. Es kam bisher noch nie vor, daß ein Reagenz weggeworfen werden mußte. Es wurde daher immer nur dann Reagenz nachgefüllt, wenn die Füllstands-Anzeige den Übergang auf das Reserve-Volumen anzeigte.

\section{Danksagung}

Die Untersuchungen wurden ż. T. durch die Fa. Greiner Electronic AG, Langenthal, unterstützt.

Für ihre wertvolle Mitarbeit danken wir Frl. M. L. Christensen, Frl. M. Lehmann und Frl. R. Takkinen.

\section{Literatur}

1. Slein, M. W., Cori, G. T. \& Cori, C. F. (1950), J. Biol. Chem. $186,763-780$.

2. Hohorst, H. J., Kreutz, F. H. \& Bücher, T. (1959), Biochem. Z. 332, 18-46.

3. Schmidt, F. (1962), Klin. W.ochenschr. 40, 585-589.

4. Barthelmai, W. \& Czok, R. (1962), Klin. Wochenschr. 40, 585-589.

5. Stork, H. \& Schmidt, F. H. (1968), Klin. Wochenschr. 46, 789-790.

6. Haeckel, R. (1970) diese Z. 8, 480-482.

7. Fonseca-Wollheim, F., da (1971), diese Z. 9; 497-502
8. Richterich, R. \& Dauwalder, H. (1971), Schweiz. Med. Wochenschr. 101, 615-618.

9. Richterich, R. (1971), Klinische Chemie, Theorie und Praxis, 3. Aufl., S. 265-278. Karger, Basel.

10. Schubert, G. E. (1964), Klin. Wochenschr. 42, 619-622.

11. Renschler, H. E., Weicker, H. \& von Baeyer, H. (1965), Deut. Med. Wochenschr. 90, 2349-2353.

12. Keller, D. M. (1965), Clin. Chem. 11, 471-477.

13. Peterson, I. J. (1968), Clin. Chem. 14, 513-520.

14. Peterson, I. J. \& Young, D. S. (1968), Analyt. Biochem. $23,301-316$. 
15. Koenig, R., Dauwalder, H. \& Richterich, R. (1971), Schweiz. Med. Wochenschr. 101, 860-866.

16. Schersten, B. \& Tibbling, G. (1968), Clin. Chem. 14, 243-252.

17. Harding, U. \& Heinzel, G. (1969), diese Z. 7, 640-643.

18. Yee, H. Y. (1972), Clin. Chem. 18, 1416-1419.

19. Neeley, W. E. (1972), Clin. Chem. 18, 509-515.

20. Haeckel, R. \& Haeckel, H. (1972), diese Z. 10, 453-461.

21. Schmitt, A., Keller, I. \& Ringhardtz, I. (1972), Beiträge zur Klinischen Chemie, Heft 8 (Bodenseewerk PerkinElmer \& Co. GmbH).

22. Greiner, R. (1973), diese Z. 11, 76-86.

23. Richterich, R. \& Greiner, R. (1971), Automated Multiphasic Health Testing, S. 124-127. Engeneering Foundation, New York.

24. Richterich, R. (1973), Ärtzl. Lab. 19, 300-305.
25. Richterich, R. \& Ehrengruber, H. (1968), Clin. Chim. Acta 22, 417-422.

26. Richterich, R. \& Küffer, H. (1972), diese Z. 11, 553-564.

27. Kornberg, A. \& Horecker, B. L. (1953), Biochemical Preparations New York: Wiley, 3, 24.

28. Pfleiderer, G., Jeckel, D. \& Wieland, T. (1956), Biochem. Z. 328, 187-194.

29. Siegel, J. M., Montgomery, B. A. \& Bock, R. M. (1959), Arch. Biochem. Biophys. 82, 288-299.

30. Richterich, R., Greiner, R. \& Küffer, H. (1973), diese Z. 11, 65-75.

31. Pastor, J., Pauli, A. M. Orinier, M., Garde, J. F., Desvenine, J. \& Dumoulin, B. (1973), Organisation des Laboratoires, Biologie prospective, (G. Siest, ed.) p. 415-423, L'expansion scientifique Française, Paris.

Dipl. Biochem. H. Küffer Chemisches Zentrallabor Inselspital

CH-3010 Bern/Schweiz 